\title{
Phylogenomic analyses of KCNA gene clusters in vertebrates: why do gene clusters stay intact? Simone Hoegg and Axel Meyer*
}

\author{
Address: Lehrstuhl für Zoologie und Evolutionsbiologie, Department of Biology, University of Konstanz, 78457 Konstanz, Germany \\ Email: Simone Hoegg - Simone.Isabel.Hoegg@uni-konstanz.de; Axel Meyer* - Axel.Meyer@uni-konstanz.de \\ * Corresponding author
}

Published: 15 August 2007

BMC Evolutionary Biology 2007, 7:139 doi:10.1/86/1471-2148-7-139

This article is available from: http://www.biomedcentral.com/147I-2/48/7//39

(C) 2007 Hoegg and Meyer; licensee BioMed Central Ltd.

This is an Open Access article distributed under the terms of the Creative Commons Attribution License (http://creativecommons.org/licenses/by/2.0), which permits unrestricted use, distribution, and reproduction in any medium, provided the original work is properly cited.
Received: 7 December 2006

Accepted: 15 August 2007

\begin{abstract}
Background: Gene clusters are of interest for the understanding of genome evolution since they provide insight in large-scale duplications events as well as patterns of individual gene losses. Vertebrates tend to have multiple copies of gene clusters that typically are only single clusters or are not present at all in genomes of invertebrates. We investigated the genomic architecture and conserved non-coding sequences of vertebrate KCNA gene clusters. KCNA genes encode shakerrelated voltage-gated potassium channels and are arranged in two three-gene clusters in tetrapods. Teleost fish are found to possess four clusters. The two tetrapod KNCA clusters are of approximately the same age as the Hox gene clusters that arose through duplications early in vertebrate evolution. For some genes, their conserved retention and arrangement in clusters are thought to be related to regulatory elements in the intergenic regions, which might prevent rearrangements and gene loss. Interestingly, this hypothesis does not appear to apply to the KCNA clusters, as too few conserved putative regulatory elements are retained.

Results: We obtained KCNA coding sequences from basal ray-finned fishes (sturgeon, gar, bowfin) and confirmed that the duplication of these genes is specific to teleosts and therefore consistent with the fish-specific genome duplication (FSGD). Phylogenetic analyses of the genes suggest a basal position of the only intron containing KCNA gene in vertebrates (KCNA7). Sistergroup relationships of $K C N A I / 2$ and KCNA3/6 support that a large-scale duplication gave rise to the two clusters found in the genome of tetrapods. We analyzed the intergenic regions of KCNA clusters in vertebrates and found that there are only a few conserved sequences shared between tetrapods and teleosts or between paralogous clusters. The orthologous teleost clusters, however, show sequence conservation in these regions.

Conclusion: The lack of overall conserved sequences in intergenic regions suggests that there are either other processes than regulatory evolution leading to cluster conservation or that the ancestral regulatory relationships among genes in KCNA clusters have been changed together with their regulatory sites.
\end{abstract}

\section{Background}

Higher phenotypic complexity of vertebrates has been often associated with a higher number of genes produced through whole genome duplications [1]. Genome projects and a deluge of sequence data showed that vertebrates often possess more than one copy of a gene or gene 
clusters [2-5] where invertebrates have only one. This observation together with synteny data, led to the formulation of the $2 \mathrm{R}$ hypothesis, which proposes two rounds of genome duplication in early vertebrate evolution [6-9]. An additional duplication event occurred in the lineage of ray-finned fish, the so-called fish-specific genome duplication (FSGD, 3R) [10-17]. While the duplicated genes are expected to be redundant in their function immediately following the duplication, their functions often diversify later $[18,19]$. Possible scenarios are that one copy evolves a new function (neofunctionalization) or the ancestral functions get subdivided between the paralogs (subfunctionalization) $[20,21]$. In most cases, however, one copy is expected to accumulate mutations that lead to a nonfunctional gene and finally to gene loss [20-23].

Clusters of genes belonging to the same gene family can give important insights into the evolutionary history of a genomic region, both in terms of gene loss events as well as for the evolution of the regulatory sequences surrounding it. The most prominent examples for this type of approach are the Hox gene clusters [24-28], a family of transcription factors that are not only arranged in uninterrupted clusters on the chromosome but are even expressed during embryogenesis according to their chromosomal order - a phenomenon called colinearity [reviewed in [29]. But also other gene clusters have been studied in this regard such as the ParaHox cluster [30](Siegel et al. submitted) and Fox clusters [31]. Both belong to other families of transcription factors with multiple cluster copies in vertebrate genomes. Less research in this respect has been performed on non-developmental genes.

We were interested if the patterns of molecular evolution that are found in Hox clusters can also be identified in other gene clusters and if it is also possible to identify conserved non-coding regions in them. KCNA genes are arranged in two uninterrupted clusters of three genes each which are located on chromosomes three and six in mouse and on chromosomes one and twelve in humans $[32,33]$. KCNA genes code for the Kv1 family of shakerrelated voltage-gated potassium $\left(\mathrm{K}^{+}\right)$channels, those consist of six transmembrane (TM) segments and, the most important part, the pore loop (P-region), which ensures ion selectivity $[34,35]$. The $\mathrm{Kv}$ channels are active as tetramers, usually heterotetramers. Sodium $\left(\mathrm{Na}^{+}\right)$and calcium $\left(\mathrm{Ca}^{+}\right)$channels, on the other hand, are monomers that consist of four linked domains, each of which is homologous to a single 6-TM $\mathrm{K}^{+}$channel [36]. Studies on the genomic organization of these genes so far have been limited to mammals. Upstream regulatory factors and potential regulatory elements have not been described previously. The number of KCNA genes and their genomic arrangement in ray-finned fish has not been studied before. Here we extend these comparative genomic approaches to other lineages of vertebrates and compare them to the situation in the genome of invertebrates.

We conducted an analysis of complete genome sequences of tetrapods and ray-finned fish KCNA genes and investigated the entire genome content when possible. In an effort to increase the database on basal fish, for which such data were not available prior to this study, we added new data using a PCR approach with universal primers and cloned the PCR products. We also included data from the non-vertebrate chordates Branchiostoma floridae and Ciona intestinalis for a better estimate of the age of this gene family. We constructed gene trees to permit inferences about the timing of the gene duplications and the cluster duplications. Furthermore, we aimed to test the hypothesis that the conservation of the genomic architecture of a gene cluster is linked to the content of conserved elements within the intergenic regions. To this end we investigated the 3-gene-cluster of KCNA genes (Kv1, shaker-related potassium channels) in several species of vertebrates. In tetrapods, two clusters exist (KCNA3KCNA2-KCNA10, KCNA6-KCNA1-KCNA5), while teleosts were found to have four clusters.

\section{Results}

Tetrapods, such as human, chicken and frog, have two three-gene-clusters (3-2-10, 6-1-5) and two additional genes, KCNA4 and KCNA7, which are located elsewhere in the genome (Figure 1). Teleost fish such as pufferfish, medaka, stickleback and zebrafish were found to have four clusters of KCNA genes. According to available results from genome sequencing projects, KCNA5a was lost. For $K C N A 7$, duplicates were found in medaka and two copies of KCNA4 are present in the osteoglossomorph elephantnose fish (Gnathonemus petersi). All of these genes are conserved in their transmembrane domains and in the poreloop region, but the other parts of these genes are highly variable and impossible to align between different members of this gene family. In the tunicate Ciona intestinalis only one KCNA gene was found while we received several BLAST hits for the amphioxus (Branchiostoma floridae) genome.

A phylogenetic analysis of all KCNA genes suggests a diversification of KCNA genes in the vertebrates and basal position of KCNA7 among them (Figure 2); it is the only vertebrate KCNA gene that contains a single intron while all others are intronless. In invertebrates, such as Ciona intestinalis and Drosophila melanogaster, the KCNA/shaker gene has multiple introns, but the position of the introns is not conserved between invertebrates and vertebrates (data not shown). Genes identified from the Branchiostoma floridae genome, however, consisted of a single exon indicating that the vertebrate KCNA7 gene acquired its 


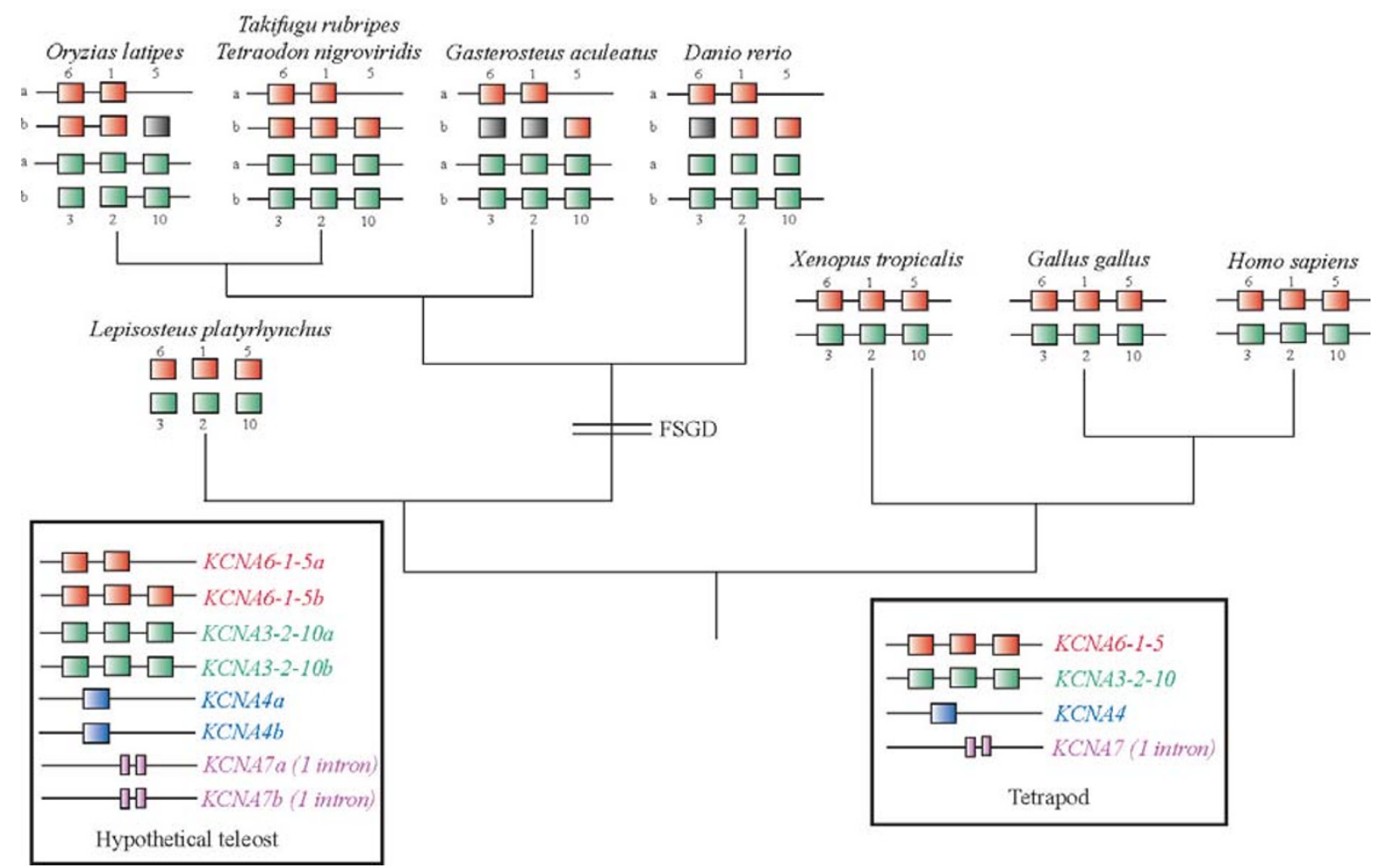

Figure I

Phylogenetic scheme of KCNA cluster evolution. Non-connected genes indicate missing linkage/genomic data. Grey squares show hypothetical genes that most likely exist, but are still missing from the current versions of genomic databases. The boxes include genes that are not part of KNCA clusters. The teleost state is hypothetical since we found duplicated KCNA4 genes in Gnathonemus petersi and duplicated KCNA7 genes in Oryzias latipes, but no teleost studied so far showed the full set of duplicated genes.

intron independently (Figure 3). The three best BLAST hits from Branchiostoma floridae with vertebrate KCNA genes were included in the phylogenetic analysis. All three genes are positioned on different scaffolds and no clusters were found. We obtained additional hits with the Branchiostoma genome, some of which also received KCNA best hits in BLAST searches against the GenBank database. However, their high sequence divergence made reliable estimation of their phylogenetic position relative to the outgroup in the KCNA gene tree impossible. The three Branchiostoma KCNA genes included in the analysis form a monophyletic group basal to the vertebrate genes, indicating a series of independent gene duplication within the amphioxus lineage.

Within the vertebrate part of the tree, KCNA5 and KCNA10 form a monophyletic group, as do KCNA1 and KCNA2 (Figure 2). This finding supports the hypothesis that the two clusters are the result of a complete duplica- tion of the original cluster rather than of independent tandem duplications, which would have phylogenetically grouped neighboring genes on chromosomes in the tree. Only the KCNA3/KCNA6 gene pair does not reflect a pattern of whole genome duplication(s).

Our PCR based approach yielded two KCNA genes from Hydrolagus colliei (spotted ratfish, KCNA2, 5), four genes from Acipenser baerii (sturgeon, KCNA1, 2, 6, 10), six genes from Lepisosteus platyrhynchus (gar, KCNA1, 2, 3, 5, 6 , 10), and nine genes from Gnathonemus petersii (elephantnose fish, KCNA1b, 2a?, 3b, 4a, 4b, 5b, 6b, 10a, $10 b$ ) [for accession numbers see Additional file 4]. We performed phylogenetic analyses of the ancient duplicates (KCNA3-6, KCNA2-1 and KCNA10-5) and used KCNA4 as outgroup [see Additional files 1, 2, 3]. KCNA4 is not part of the clusters, but phylogenetically closely related without obvious acceleration of evolutionary rates (Figure 2). In this way, we avoid reconstruction artifacts due to a too 


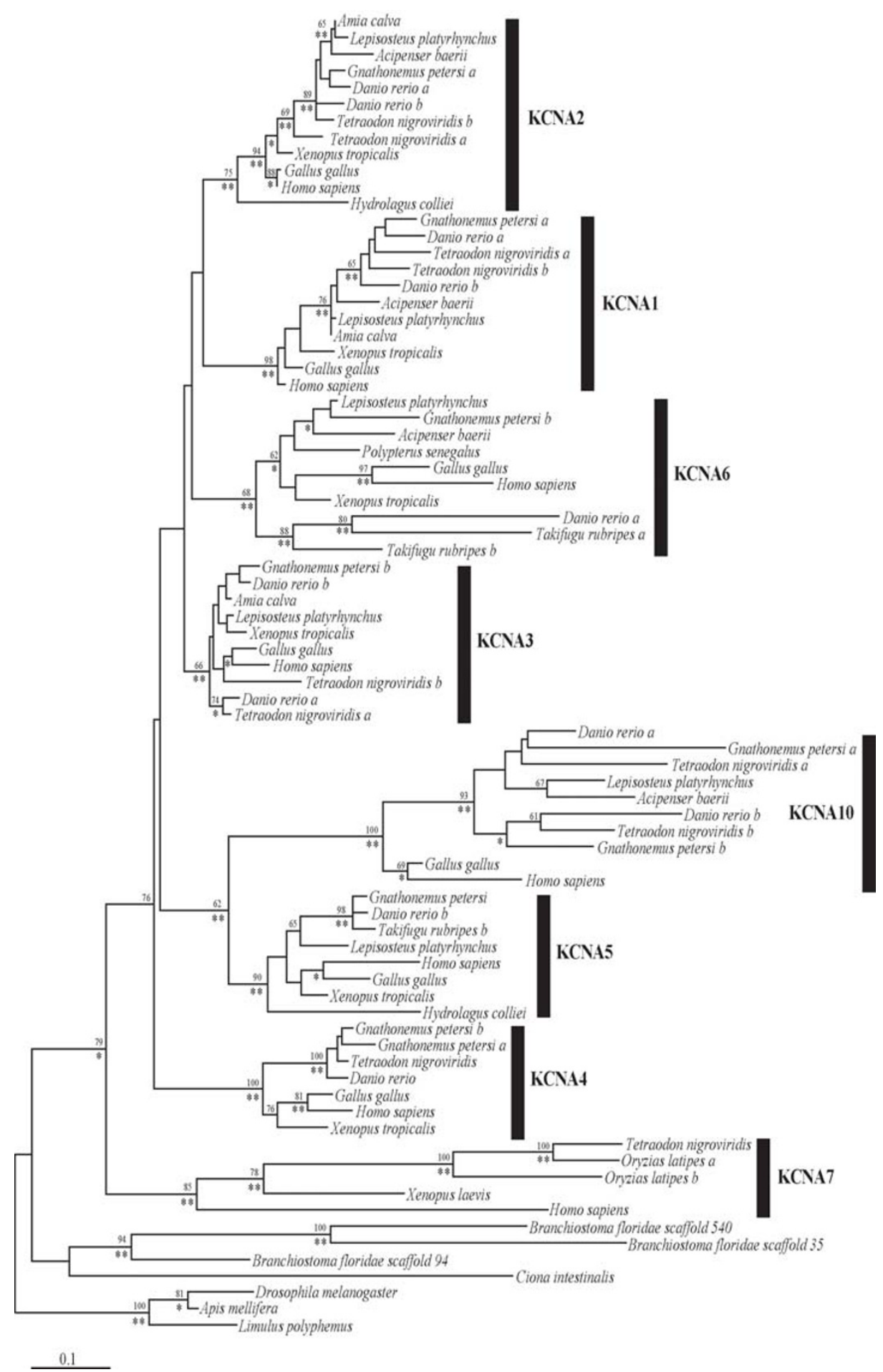

Figure 2

Maximum likelihood tree of KCNA gene family based on 80 sequences and 364 amino acid positions. The tree was obtained using PhyML [5], with 500 bootstrap replicates, values are shown by the first numbers. Posterior probabilities as obtained by MrBayes 3.I.I [59] (100 000 generations) are indicated with asterisks. (** $=100 \%$ PP, * $=99-95 \%$ PP) 


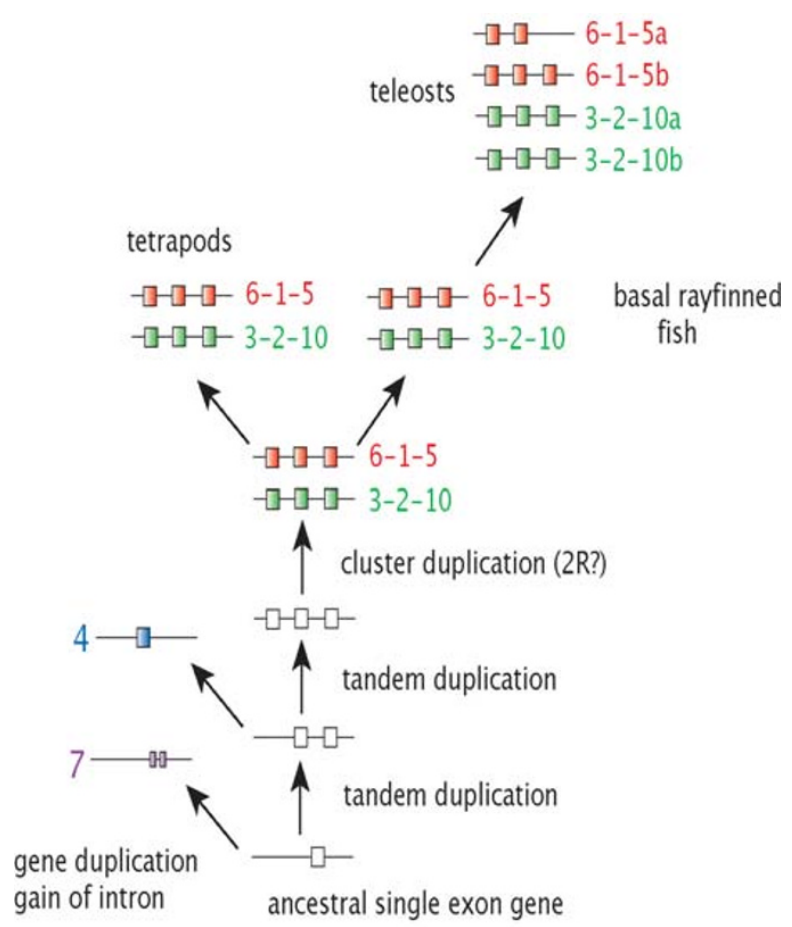

\begin{abstract}
Figure 3
Proposed scenario for the evolution of KCNA genes and clusters in vertebrates. Based on our analyses we suggest that all KCNA genes are derived from an ancestral intronless gene, as all genes included from Branchiostoma floridae are intronless and that KCNA7 in vertebrates independently gained an intron. Two tandem duplications led to the three gene clusters found in today's genomes, which was probably duplicated initially before the origin of the gnathostomes. Probably this is linked to the second genome duplication $(2 \mathrm{R})$ during vertebrate evolution. The four clusters in teleost fish originated through the fish-specific genome duplication (FSGD, 3R).
\end{abstract}

divergent outgroup as would expected be with the ancestral KCNA7 gene.

Our KCNA3/6 dataset encompassed 42 sequences and included sequences from human, chick and frog (378 amino acid positions) and resulted in Maximum Likelihood and Bayesian inference trees which were congruent for the well supported nodes and showed only minor differences within the not strongly resolved parts of the tree [see Additional file 1]. While the KCNA3 genes were phylogenetically separate from the KCNA6 genes, the resolution within each of these sets of orthologous genes is poor and the relationships, especially among the fish paralogous groups, could not be identified with confidence. The assignment into "a" and "b" paralogs was done based on the position in the clusters when genomic data was available, and the newly determined orthologous KCNA genes for the fish were assigned names accordingly. The evolutionary rates differ clearly between the orthologous groups (KCNA3 vs. KCNA6) as well as between the fishspecific a- and b-paralogs.

The KCNA2/1 analysis shows also a clear division between these two set of genes, but there are no obvious rate differences between them, only within the teleosts somewhat increased rates are apparent [see Additional file 2]. For both genes, the non-teleost fish sequences (Acipenser, Amia, Lepisosteus) are pro-orthologous of the FSGD as had been proposed in previous studies [10,37,38]. Studies based on Hox genes as well as other nuclear genes (sox11, tyrosinase, $f z d 8, P O M C$ ) found a phylogenetic timing of the FSGD after the divergence of Polypteriformes (bichir), Acipenseriformes (sturgeons), Lepistosteidae (gar) and Amiidae (bowfin), but before the teleost radiation including the most basal group of the Osteoglossiformes $[10,30]$. The Gnathonemus KCNA2 sequence is positioned basal to the duplication in the gene tree. The Gnathonemus KCNA1 sequence is grouped with the b-paralog in the KCNA1 gene tree. But also in this analysis, statistical support for most of the nodes is lacking.

The KCNA5/10 tree shows a similar pattern as the KCNA3/ 6 analysis with a clear acceleration of evolutionary rates in the KCNA10 genes, a trend that is even more pronounced in ray-finned fish [see Additional file 3]. The Hydrolagus KCNA5 sequence is phylogenetically grouped with the other KCNA5 genes with good phylogenetic support $(94 \% \mathrm{BP}, 100 \% \mathrm{PP})$, indicating that the duplication of the clusters leading to KCNA5 and KCNA10 occurred before the divergence of cartilaginous fish as previously proposed [39].

Even though the phylogenetic analyses of KCNA genes cannot pinpoint the duplication event in the fish phylogeny with a high degree of certainty, the numbers of identified genes implies that the phylogenetic split of basal lineages that include Acipenser, Lepisosteus and Amia from the fish stem lineage precedes the duplication event, while duplicated KCNA4 and KCNA10 genes suggest that Osteoglossomorphs (Gnathonemus petersi) diverged after the 3R event. This interpretation is in agreement with previous analyses on the phylogenetic timing of the FSGD [10].

We analyzed the non-coding regions of the complete clusters using the Tracker software [40], which detects clusters of such phylogenetic footprints (putative transcription factor binding sites) termed cliques (FC). Following the definition of phylogenetic footprints as in Tagle et al. [41], we only compared sequences with an additive evolution- 
Table I: Pairwise comparison of KCNA 6-I-5 clusters Above the diagonal are the numbers of shared cliques (clusters of phylogenetic footprints) based on Tracker analyses; below are the complete lengths of shared elements. Excluded direct comparisons between pufferfish clusters are printed in bold. "a" and "b" refer to the duplicated fish clusters.

\begin{tabular}{|c|c|c|c|c|c|c|c|c|c|}
\hline 615 & $H_{s}$ & $G g$ & $X t$ & Tra & Tna & Ola & Gaa & Dra & $T n b$ \\
\hline Hs & - & 45 & 25 & 7 & 7 & 7 & 5 & 5 & 5 \\
\hline $\mathrm{Gg}$ & 2959 & - & 30 & 10 & 5 & 8 & 5 & 5 & 5 \\
\hline$X t$ & $|87|$ & 2100 & - & 6 & 5 & 6 & 4 & 4 & 3 \\
\hline Tra & 528 & 622 & 345 & - & 17 & 19 & 17 & 7 & 2 \\
\hline Tna & 517 & 378 & 311 & 2340 & - & 16 & 11 & 6 & 1 \\
\hline Ola & 571 & 513 & 370 & 2354 & 1808 & - & 16 & 7 & 0 \\
\hline Gaa & 214 & 200 & 218 & 1717 & 1553 & 1815 & - & 7 & 0 \\
\hline Dra & 244 & 173 & 205 & 1245 & 1212 & 1358 & 1039 & - & 0 \\
\hline$T n b$ & 195 & 221 & 140 & 102 & 40 & 0 & 0 & 0 & - \\
\hline
\end{tabular}

Abbreviations are standing for human $(\mathrm{Hs})$, chicken $(\mathrm{Gg})$, frog $(\mathrm{Xt})$, zebrafish $(\mathrm{Dr})$, stickleback $(\mathrm{Ga})$, medaka $(\mathrm{Ola})$, and the two pufferfishes $(\mathrm{Tn}$, $\mathrm{Tr})$.

ary time of at least 250 million years, i.e. species that diverged at least 125 million years ago, and therefore we excluded comparisons of orthologous pufferfish clusters $[42,43]$. For the KCNA 6-1-5 comparisons, we included the partial Tetraodon KCNA 5-1b cluster, since it was currently the only available genomic sequence for this paralogon. Because of a large sequence gap in the intergenic region of the medaka KCNA $6-1 b$, we omitted this sequence from the analysis. We also could not include the Danio KCNA 3-2-10a cluster, since no linkage information for KCNA2a was available in the current assembly. For these analyses we were able to include a total of 20 clusters (11KCNA 3-2-10, 9 KCNA 6-1-5 and we obtained 670 FC of which 182 are shared by more than two species. The alignments and positions of those are given in the Additional file 5. Since an analysis using untreated sequences resulted an unusual high number of cliques shared only between the two paralogous human clusters as well as between the two Xenopus clusters, an effect probably due to the abundance of repetitive elements, we used RepeatMasker to remove those elements and repeated the analyses [44]. This strategy reduced the number of intra-species hits successfully.

We also analyzed the number of FCs and the length of conserved sequences between orthologous and paralogous clusters. Between orthologous clusters, the number of conserved elements follows the expected patterns, at least within tetrapods and the orthologous fish clusters respectively (Tables 1,2). Paralogous fish KCNA-gene clusters share surprisingly few conserved elements.

Interesting in this regard is the comparison between paralogous clusters (e.g. Homo 3-2-10 vs. Homo 6-1-5). While one might expect to find a reduced number of conserved sequences compared to the orthologous comparisons (e.g. Homo 3-2-10 vs. Xenopus 3-2-10), this is not the case for comparisons among tetrapods. The human KCNA3-2-
10 cluster shares more FCs with its paralog KCNA6-1-5 than with the more closely related frog KCNA3-2-10 cluster, even after the elimination of repetitive sequences (Table 3). This effect is mainly due to relatively short FCs that are found only in the human sequences and not in any other species.

\section{Discussion}

Up to now, shaker-related voltage-gated potassium channels have been mainly studied in tetrapods with a strong emphasis on functional and structural aspects $[34,35,45]$, but not within a larger phylogenomic framework. Neither the number of genes within ray-finned fish nor the phylogenetic relationships of these genes have been studied previously. Yet, this information clearly provides useful insights for the comparison of experimental and functional studies. With prior knowledge of the existence of two 3-gene-clusters (3-2-10 and 6-1-5) in mammals $[32,33]$, we were able identify two clusters in chicken and the frog Xenopus tropicalis as well. The human clusters are positioned on chromosomes (chr 1, chr 12) that have been reported to contain a number of genes duplicated during large scale duplication events [7]. The addition of new data from fish reveals the existence of four clusters in teleosts as a result of the fish-specific genome duplication (FSGD). KCNA3-2-10 cluster of Tetraodon are on chromosomes 11 and 9, in Oryzias on chromosomes 7 and 5. The origin of those chromosomes through the FSGD has been proposed previously $[16,46]$. Because of missing linkage data, similar conclusions for the KCNA6-1-5 clusters cannot be drawn. Due to a lack of data from lampreys and hagfish, the timing of the first cluster duplication (leading to the two-cluster situation in tetrapods) is unknown (Figure 1). Most likely their origin is the result of one of the genome duplication events during chordate/vertebrate evolution $(2 \mathrm{R})[7,47]$. The two genes we identified from Hydrolagus colliei (spotted ratfish, Chondrichthyes) so far can unambiguously be assigned to their tetrapod 
Table 2: Pairwise comparison of KCNA 3-2-10 clusters Above the diagonal are the numbers of shared cliques (clusters of phylogenetic footprints) based on Tracker analyses; below are the complete lengths of shared elements. Excluded direct comparisons between pufferfish clusters are printed in bold. "a" and "b" refer to the duplicated fish clusters.

\begin{tabular}{|c|c|c|c|c|c|c|c|c|c|c|c|}
\hline 3210 & Hs & $G g$ & $X t$ & Gaa & Ola & Tra & Tna & Trb & $T n b$ & $G a b$ & Drb \\
\hline Hs & - & 28 & 24 & 5 & 6 & 5 & 5 & 8 & 6 & 13 & 10 \\
\hline$G g$ & 1788 & - & 16 & 9 & 7 & 3 & 6 & 8 & 4 & 6 & 6 \\
\hline$X t$ & 1375 & 1077 & - & 10 & 13 & 6 & 10 & 11 & 8 & 11 & 12 \\
\hline Gaa & 450 & 514 & 507 & - & 47 & 41 & 39 & 8 & 8 & 7 & 9 \\
\hline Ola & 445 & 462 & 618 & 4560 & - & 37 & 34 & 4 & 5 & 4 & 7 \\
\hline Tra & 484 & 282 & 395 & 4129 & 3599 & - & 33 & 3 & 6 & 4 & 5 \\
\hline Tna & 412 & 452 & 497 & 4270 & 3520 & 3052 & - & 6 & 8 & 9 & 6 \\
\hline$T r b$ & 559 & 408 & 553 & 827 & 460 & 444 & 505 & - & 55 & 75 & 10 \\
\hline$T n b$ & 439 & 303 & 414 & 1005 & 586 & 703 & $74 I$ & 4966 & - & 70 & 8 \\
\hline Gab & 730 & 370 & 550 & 803 & 460 & 493 & 647 & 5952 & 5527 & - & 8 \\
\hline Drb & 788 & 469 & 602 & 805 & 586 & 600 & 574 & 980 & 813 & 906 & - \\
\hline
\end{tabular}

Abbreviations are standing for human $(\mathrm{Hs})$, chicken $(\mathrm{Gg})$, frog $(\mathrm{Xt})$, zebrafish $(\mathrm{Dr})$, stickleback $(\mathrm{Ga})$, medaka (Ola), and the two pufferfishes $(\mathrm{Tn}$, Tr).

orthologs (KCNA2, KCNA5). This finding implies that sharks already possesses the two KCNA clusters [39], the timing of the duplication with respect to the cyclostomes remains unclear. The KCNA complement of the cephalochordate Branchiostoma floridae shows an independent series of gene duplication, with all genes being intronless. Thus cluster formation in the vertebrate lineage occurred after the divergence with the cephalochordates. In Ciona intestinalis, a tunicate, only one KCNA sequence was found, which consisted of at least five exons, of which only four were identified unambiguously. The amino-terminus was found to be highly variable among different vertebrate genes and thus BLAST searches with invertebrate sequences yielded no hits for the amino terminus.

The phylogenetic analysis suggested KCNA7 as the most basal vertebrate KCNA gene. This gene has two exons, while the rest of the vertebrate genes are intronless (Figure 1 ). The phylogenetic analyses could not resolve all sistergroup relationships between the intronless KCNA genes (KCNA1-6, 10) with high confidence. This might be due to the extreme rate difference among the various members of this gene family as well as, the rapid succession of duplication events. We propose an evolutionary scenario of two consecutive tandem duplications that formed a first cluster. Mostly likely during the $2 \mathrm{R}$ genome duplication, the entire cluster was duplicated leading to the situation found in tetrapods (Figure 3 ). This hypothesis is also supported by the sistergroup relationship found between KCNA5 and 10, as well as between KCNA1 and 2 genes, which are now parts of different KCNA clusters. For the other gene pairs, the data are not as clear, but the alternative scenario of independent tandem duplications on different chromosomes is not supported by the topology of the tree. We suppose that the reconstruction problems are caused by the fast evolution of KCNAG as well as by the short basal branches that render phylogenetic reconstructions difficult. This also implies that the tandem duplications must have happened, evolutionarily speaking, only shortly before the entire cluster duplications. The origin of the KCNA4 gene from the KCNA7 gene progenitor could be the result of a large-scale duplication (1R) event followed by the loss of several genes but currently there are no synteny data available to add support this hypothesis.

The phylogenetic analyses, also with smaller datasets [see Additional files 1, 2, 3], are hampered by pronounced rate differences between paralogs and increased rates of evolution of KCNA paralogs in the teleost fish. Accelerated rates of evolution in some fish genes have been observed before [48-50]. Pronounced rate differences can lead to wrong topologies [51]. An accelerated rate of evolution might be due to reduced selective pressure because of gene redundancy after duplication, or, alternatively, might be due to positive Darwinian selection associated with a change in function. Yet, currently, a link between expression and the formation of heterodimers between different subtypes and their evolutionary rates is not obvious. More functional data with respect to gene expression and its regulation, as well as the formation of heteromers from more species, especially teleost fishes, could provide further insights. To date, expression data are available only for few tetrapod species such as mouse, human and chicken. Furthermore, these studies only examined expression differences in a subset of tissue types [52].

For a better understanding of the characteristics of the gene clusters, we first performed an alignment based VISTA plot analysis [53]. However, no clear conserved regions across all species included were apparent, only between the orthologous fish clusters (results not shown). For a more detailed analysis, we ran the Tracker program, 
Table 3: Pairwise comparisons between paralogous clusters and the number of shared PFCs (phylogenetic footprint cliques) and their complete lengths

\begin{tabular}{|c|c|c|c|c|c|c|c|c|c|c|c|c|}
\hline \multirow{2}{*}{$\begin{array}{c}\text { KCNA } \\
6-1-5 \\
\text { clusters }\end{array}$} & \multicolumn{12}{|c|}{ KCNA 3-2-10 clusters } \\
\hline & & Hs & $G g$ & $X t$ & Gaa & Ola & Tra & Tna & $\operatorname{Trb}$ & $T n b$ & $G a b$ & Drb \\
\hline & \multicolumn{12}{|c|}{ Number of cliques } \\
\hline & Hs & 24 & 17 & 21 & 13 & 13 & 9 & 7 & 8 & 7 & 13 & 13 \\
\hline & $\mathrm{Gg}$ & 20 & 12 & 20 & 9 & 18 & 9 & 6 & 15 & 9 & 15 & 8 \\
\hline & $X t$ & 17 & 11 & 39 & 8 & 18 & 11 & 10 & 12 & 6 & 12 & 11 \\
\hline & Tra & 3 & 2 & 3 & I & 2 & 2 & 1 & 3 & I & 3 & 2 \\
\hline & Tna & 3 & 3 & 3 & I & 2 & I & I & 3 & 2 & 2 & 2 \\
\hline & Ola & I & 2 & 6 & 1 & 2 & I & I & 3 & 1 & 2 & 2 \\
\hline & Gaa & 3 & I & 2 & I & 2 & I & I & 2 & I & I & 1 \\
\hline & Dra & 2 & 1 & 0 & I & 1 & 1 & 1 & 2 & I & 0 & 2 \\
\hline & $T n b$ & 2 & 2 & 2 & 2 & 1 & 2 & 2 & 2 & I & 2 & 3 \\
\hline & \multicolumn{12}{|c|}{ Total length } \\
\hline & Hs & 1248 & 774 & 1177 & 564 & 584 & 407 & 340 & 644 & 338 & 830 & 556 \\
\hline & $\mathrm{Gg}$ & 980 & 508 & 946 & 420 & 815 & 382 & 293 & 869 & 381 & 987 & 330 \\
\hline & $X t$ & 801 & 450 & 3014 & 332 & 742 & 521 & 438 & 633 & 302 & 639 & 406 \\
\hline & Tra & 155 & 444 & 512 & 33 & 397 & 89 & 33 & 214 & 38 & 163 & 454 \\
\hline & Tna & 155 & 510 & 486 & 43 & 421 & 43 & 43 & 139 & 69 & 89 & 359 \\
\hline & Ola & 136 & 477 & 667 & 66 & $44 I$ & 66 & 66 & 289 & 60 & 196 & 427 \\
\hline & Gaa & 100 & 58 & 98 & 99 & 132 & 99 & 99 & 114 & 31 & 31 & 291 \\
\hline & Dra & 83 & 21 & 0 & 52 & 52 & 52 & 52 & 127 & 42 & 0 & 105 \\
\hline & $T n b$ & 50 & 57 & 64 & 68 & 72 & 74 & 67 & 54 & 23 & 79 & 98 \\
\hline
\end{tabular}

which is based on an initial BlastZ algorithm [40]. We performed a complete analysis using 20 sequences and obtained $670 \mathrm{FC}$, of which 182 consist of more than two sequences. Alignments and sequence positions of the FCs are provided in the supplementary data. The percentage of FCs of the complete sequences was very low in tetrapods but the teleost clusters showed comparable values (11$34 \%$ ) to the Hox clusters (10-38\%, Hoegg et al, submitted) (Table 4). The lack of signal observed in Vista plots is rather a pronounced lack of elements conserved between tetrapods and fish. Hox genes as developmental key factors, on the other hand, are involved in many different pathways and, therefore, have more regulatory elements that are conserved over long evolutionary distances. Expression of KCNA genes, on the other hand, was studied so far only in adult tissues, but a more continuous expression pattern in specific tissues (e.g. brain, heart, muscle) might be expected. Since KCNA genes are active as homo- and heterotetramers, expressional "fine tuning" might also be accomplished through the expression of a combination of different KCNA genes at different stages during development and in different tissue types. The analyses of orthologous clusters revealed the expected pat- tern for the tetrapods and within orthologous fish clusters, i.e., more closely related organisms share more cliques, while the paralogous fish clusters share less conserved elements (Tables 1,2). This finding again is most likely caused by faster evolution in duplicated fish clusters.

However, the comparisons between paralogous clusters of the same species showed an unexpected pattern for the tetrapods included in this study (Table 3 ). The phylogenetic analyses show that the clusters were duplicated before the tetrapod - ray-finned fish split (+/- 450 million years ago [54]). Using sequences that were not "masked" for repetitive sequences in the Tracker analyses as proposed in the original publication [40], leads to high numbers of FCs shared only between paralogous sequences of one tetrapod species. The teleost KCNA clusters are more compact, contain less repetitive sequences and are therefore less susceptible to false hits. For gnathostome Hox clusters, where selection is acting against repetitive sequences [55], masking can be neglected, but for other gene clusters it can be useful. 
Table 4: Length of the sequences in different organisms, counted from the start codon of the 5 'most gene to the stop of the 3'most gene or until the next gene The second column contains the complete length of FCs from on sequence, and its percentage of the complete length of the cluster.

\begin{tabular}{|c|c|c|c|}
\hline Sequence & Total length & $\mathrm{Bp}$ in cliques & $\%$ of bp cliques \\
\hline Homo 3-2-10 & $|5740|$ & 5555 & 3.5 \\
\hline Gallus 3-2-10 & 54669 & 4149 & 7.6 \\
\hline Xenopus 3-2-10 & 154969 & 7587 & 5.9 \\
\hline Gasterosteus 3-2-10 a & 30201 & 6675 & 22.1 \\
\hline Oryzias 3-2-10 a & 48863 & 6405 & 13.1 \\
\hline Tetraodon 3-2-10 a & 24581 & 4403 & 17.9 \\
\hline Takifugu 3-2-10 a & 26792 & 4680 & 17.5 \\
\hline Tetraodon 3-2-10 b & 23872 & 6137 & 25.7 \\
\hline Takifugu $3-2-10 \mathrm{~b}$ & 26629 & 6815 & 25.6 \\
\hline Gasterosteus 3-2-10 b & 30044 & 8186 & 27.2 \\
\hline Danio 3-2-10 b & 88550 & 3091 & 3.5 \\
\hline Homo 6-I-5 & 237607 & 7497 & 3.2 \\
\hline Gallus 6-I-5 & 173038 & 7279 & 4.2 \\
\hline Xenopus 6-1-5 & | 44682 & 7518 & 5.2 \\
\hline Tetraodon 6-I a & 8433 & 2044 & 24.2 \\
\hline Takifugu 6-la & 10347 & 2920 & 28.2 \\
\hline Oryzias 6-1a & 8252 & 2835 & 34.4 \\
\hline Gasterosteus 6-I a & 7038 & 1892 & 26.9 \\
\hline Danio 6-I a & 9756 & 1119 & 11.5 \\
\hline Tetraodon I-5 b & 10689 & 1176 & 11.0 \\
\hline
\end{tabular}

This finding might imply that the clustered structure of KCNA genes is to some extent due to common expression domains of the genes within each cluster [56], or at least that the regulatory elements are not conserved over larger evolutionary distances. Since expression data is not widely available especially not for non-mammal species, it is difficult to draw further conclusions about this testable hypothesis.

\section{Conclusion}

The KCNA gene family underwent a series of duplications within the vertebrates leading to eight genes in tetrapods and more than 13 in teleosts. The initial gene underwent at least two tandem duplications forming two three-geneclusters, that were most likely duplicated during a genome duplication before the divergence of the Chondrichthyes. The molecular evolutionary analysis of the KCNA gene clusters showed only few footprint cliques (FC) that are conserved over large evolutionary distances, while among the teleost clusters more conservation was evident. Shared regulatory elements do not seem to be the major force that keep these clusters intact and therefore do not pose a general rule for gene cluster retention.

\section{Methods}

\section{Database searches}

Complete clusters of KCNA genes were downloaded from public databases such as Genbank (Homo sapiens), Ensemble (Gallus gallus, Gasterosteus aculeatus, Oryzias latipes,
Danio rerio), JGI (Branchiostoma floridae, Xenopus tropicalis, Takifugu rubripes), and Genoscope (Tetraodon nigroviridis).

\section{Amplification of KCNA genes}

Universal primers (KCNA.uni.F270.super ATY YTN TAY TAY TAY CAR TCI GGI GG, KCNA.uni.R583.super ACN GTN GTC ATN GRI ACI GCC ACC A) were designed based on known sequences. These primers amplified all intronless KCNA genes (KCNA 1, 2, 3, 4, 5, 6, 10) in vertebrates. PCRs were performed in $25 \mu \mathrm{l}$ reactions using $0.5 \mu \mathrm{l}(1$ Unit $/ \mu \mathrm{L}$ ) of REDTaq DNA polymerase (Sigma), $2.5 \mu \mathrm{l}$ $10 \times$ REDTaq PCR reaction buffer, $1.5 \mu \mathrm{l}$ dNTPs $(2.5 \mathrm{mM}$ each), $1.0 \mu \mathrm{MgCl}_{2}(25 \mathrm{mM}), 1.0 \mu \mathrm{l}$ of each primer (10 $\mu \mathrm{M})$ and $20 \mathrm{ng}$ of genomic DNA. 35 PCR cycles with an annealing temperature of $50^{\circ} \mathrm{C}$ and an extension time of two minutes were conducted in each experiment. PCRs were performed with DNA from Hydrolagus colliei (spotted ratfish, Chondrichthyes) Polypterus senegalus (bichir), Acipenser baerii (Siberian sturgeon), Lepisosteus platyrhynchus (Florida gar), Amia calva (bowfin), Gnathonemus petersi (elephant nose fish) and Oreochromis niloticus (tilapia).

PCR fragments were subcloned using the TOPO-TA cloning kit (Invitrogen). Colony PCRs were performed followed by sequencing on an ABI-Hitachi 3100 capillary sequencer following the manufacturer's instructions using the BigDye Terminator cycle-sequencing ready reaction kit (Applied Biosystems Inc.). 
Sequences were assembled and checked using Sequence Navigator ${ }^{\mathrm{TM}} 1.0$ (Applied Biosystems). Accession numbers and genomic locations of sequences used in this study are listed in Additional file 4.

\section{Phylogenetic analyses}

Deduced amino acid sequences were aligned with ClustalW and manually refined in BioEdit [57]. Amino- and carboxy-terminal sequences were not alignable and therefore were excluded from further analyses. Models of sequence evolution were chosen with ProtTest [58] using the AIK criterion. Maximum likelihood analyses were performed with PhyML [59], running 500 bootstrap replicates. Bayesian inference were performed in MrBayes3.1 [60] for 100000 generations, running 4 chains, sampling every $10^{\text {th }}$ tree and a burnin value of 5000 .

\section{Cluster analyses}

Complete gene clusters from Homo sapiens, Gallus gallus, Xenopus tropicalis, Takifugu rubripes, Tetraodon nigroviridis, Gasterosteus aculeatus, Oryzias latipes and Danio rerio were analyzed using the Tracker program [40] for the identification of conserved sequences. Since suspicious amounts of paralogous hit from one species were found for human and frog, we removed repetitive sequences using RepeatMasker [44]. We excluded direct comparisons between the two pufferfish sequences to avoid biased results due to their recent common ancestry. Footprint cliques with more than two sequences are given in Additional file 5. We also performed VISTA plots using LAGAN multiple alignments [53].

\section{Authors' contributions}

SH participated in the design of the study, carried out lab work and drafted the manuscript. AM participated in the design of the study and the writing of the manuscript.

\section{Additional material}

\section{Additional file 1}

Maximum likelihood tree of KCNA3/6. The dataset included 42 species of which ten were outgroup sequences (KCNA4) and had a total length of 378 amino acid positions. The model applied was JTT $+I+G$ (pinv $=$ $0.35, a=0.61)$. Values in the front are bootstrap percentages as obtained from 500 bootstrap replicates. Posterior probabilities as obtained by MrBayes 3.1.1 [59](100 000 generations) are indicated with asterisks. $\left(^{* *}=100 \% P P,{ }^{*}=99-95 \% P P\right)$

Click here for file

[http://www.biomedcentral.com/content/supplementary/14712148-7-139-S1.jpeg]

\section{Additional file 2}

Maximum Likelihood tree of KCNA1/2. The dataset included 49 species of which ten were outgroup sequences (KCNA4) and had a total length of 449 amino acid positions. The model applied was JTT $+I+G($ pinv $=$ $0.37, a=0.61$ ). Values in the front are bootstrap percentages as obtained from 500 bootstrap replicates. Posterior probabilities as obtained by MrBayes 3.1.1 [59](100 000 generations) are indicated with asterisks. $\left({ }^{*}=100 \% P P, *+99-95 \% P P\right)$

Click here for file

[http://www.biomedcentral.com/content/supplementary/14712148-7-139-S2.jpeg]

\section{Additional file 3}

Maximum likelihood tree of KCNA5/10. The dataset included 39 species of which ten were outgroup sequences (KCNA4) and had a total length of 360 amino acid positions. The model applied was JTT $+I+G$ (pinv $=0.42, a=0.81$ ). Values in the front are bootstrap percentages as obtained from 500 bootstrap replicates. Posterior probabilities as obtained by MrBayes 3.1.1 [59](100 000 generations) are indicated with asterisks. $\left({ }^{*}=100 \% P P,{ }^{*}=99-95 \% P P\right)$

Click here for file

[http://www.biomedcentral.com/content/supplementary/14712148-7-139-S3.jpeg]

\section{Additional file 4}

Accession numbers of nucleotide sequences, including those newly determined for this study that were analyzed in this study.

Click here for file

[http://www.biomedcentral.com/content/supplementary/1471-

2148-7-139-S4.doc]

\section{Additional file 5}

List of all footprint cliques (FCs) with more than two sequences as obtained by Tracker. For each clique, the relative position in regards to genes is given as well as the nucleotide position within the sequences. Click here for file

[http://www.biomedcentral.com/content/supplementary/14712148-7-139-S5.doc]

\section{Acknowledgements}

We thank Sonja Prohaska and Peter F. Stadler for help with the Tracker analyses. Christine Baderschneider helped with the lab work. We thank the reviewers for their constructive comments on earlier versions of this manuscript. This work was financially supported by grants of the Deutsche Forschungsgemeinschaft and the University of Konstanz to A.M. and a grant of the Landesgraduiertenförderung Baden-Württemberg to $\mathrm{S}$. $\mathrm{H}$

\section{References}

I. Ohno S: Evolution by gene duplication. New York: Springer-Verlag; 1970.

2. Ohno S: Gene duplication and the uniqueness of vertebrate genomes circa 1970-1999. Cell Dev Biol 1999, 10:517-522.

3. Meyer A, Schartl M: Gene and genome duplications in vertebrates: the one-to-four (-to-eight in fish) rule and the evolution of novel gene functions. Curr Opin Cell Biol 1999, I I:699-704.

4. Furlong RF, Holland PWH: Were vertebrates octoploid? Phil Trans R Soc Lond B Biol Sci 2002, 357:531-544.

5. Garcia-Fernandez J, Holland PW: Archetypal organization of the amphioxus Hox gene cluster. Nature 1994, 370:563-566.

6. Holland PW: More genes in vertebrates? J Struct Funct Genomics 2003, 3:75-84.

7. Dehal $\mathrm{P}$, Boore $\mathrm{JL}$ : Two rounds of whole genome duplication in the ancestral vertebrate. PLOS Biol 2005, 3:e3।4.

8. Spring J: Vertebrate evolution by interspecific hybridisation-are we polyploid? FEBS Lett 1997, 400:2-8. 
9. Lundin L-G: Gene duplications in early metazoan evolution Cell Dev Biol 1999, 10:523-530.

10. Hoegg S, Brinkmann H, Taylor JS, Meyer A: Phylogenetic timing of the fish-specific genome duplication correlates with the diversification of teleost fish. J Mol Evol 2004, 59:190-203.

II. Malaga-Trillo E, Meyer A: Genome duplications and accelerated evolution of Hox genes and cluster architecture in teleost fishes. Am Zool 200I, 4I:676-686.

12. Taylor JS, Braasch I, Frickey T, Meyer A, Van de Peer Y: Genome duplication, a trait shared by 22,000 species of ray-finned fish. Genome Res 2003, 13:382-390.

13. Taylor JS, Van de Peer Y, Braasch I, Meyer A: Comparative genomics provides evidence for an ancient genome duplication event infish. Phil Trans $R$ Soc Lond B Biol Sci 200I, 356:166I-I679.

14. Amores A, Force A, Yan YL, Joly L, Amemiya C, Fritz A, Ho RK, Langeland J, Prince V, Wang YL, Westerfield M, Ekker M, Postlethwait $\mathrm{JH}$ : Zebrafish hox clusters and vertebrate genome evolution. Science 1998, 282: I7|I-|7|4.

15. Amores A, Suzuki T, Yan Y-L, Pomeroy J, Singer A, Amemiya C, Postlethwait $\mathrm{HH}$ : Developmental roles of pufferfish hox clusters and genome evolution in ray-fin fish. Genome Res 2004, 1 4: I- 10.

16. Jaillon O, Aury J-M, Brunet F, Petit J-L, Stange-Thomann N, Mauceli E, Bouneau L, Fischer C, Ozouf-Costaz C, Bernot A, Nicaud S, Jaffe D, Fisher S, Lutfalla G, Dossat C, Segurens B, Dasilva C, Salanoubat M, Levy M, Boudet N, Castellano S, Anthouard V, Jubin C, Castelli V, Katinka M, Vacherie B, Biemont C, Skalli Z, Cattolico L, Poulain J, et al:: Genome duplication in the teleost fish Tetraodon nigroviridis reveals the early vertebrate proto-karyotype. Nature 2004, 431 :946-957.

17. Stellwag EJ: Hox gene duplication in fish. Semin Cell Dev Biol 1999, 10:53I-540.

18. Postlethwait J, Amores A, Cresko W, Singer A, Yan YL: Subfunction partitioning, the teleost radiation and the annotation of the human genome. Trends Genet 2004, 20:48I-490.

19. Hurley I, Hale ME, Prince VE: Duplication events and the evolution of segmental identity. Evol Dev 2005, 7:556-567.

20. Force A, Lynch M, Pickett FB, Amores A, Yan YL, Postlethwait ]: Preservation of duplicate genes by complementary, degenerative mutations. Genetics 1999, I5 I:153 |-1545.

21. Lynch M, Force A: The probability of duplicate gene preservation by subfunctionalization. Genetics 2000, 154:459-473.

22. Force A, Shashikant C, Stadler P, Amemiya CT: Comparative genomics, cis-regulatory elements, and gene duplication. Methods Cell Biol 2004, 77:545-56I.

23. Van de Peer Y, Taylor JS, Braasch I, Meyer A: The ghost of selection past: rates of evolution and functional divergence of anciently duplicated genes. J Mol Evol 200I, 53:436-446.

24. McGinnis W, Krumlauf R: Homeobox genes and axial patterning. Cell 1992, 68:283-302.

25. Hoegg S, Meyer A: Hox clusters as models for vertebrate genome evolution. Trends Genet 2005, 2 1:421-424.

26. Garcia-Fernandez J: The genesis and evolution of homeobox gene clusters. Nat Rev Genet 2005, 6:88I-892.

27. Meyer A, Van de Peer Y: From 2R to 3R: evidence for a fish-specific genome duplication (FSGD). Bioessays 2005, 27:937-945

28. Vandepoele K, De Vos W, Taylor JS, Meyer A, Van de Peer Y: Major events in the genome evolution of vertebrates: Paranome age and size differs considerably between ray-finned fishes and land vertebrates. Proc Natl Acad Sci USA 2004, 10 I: I 638-1643.

29. Carroll SB, Grenier JK, Weatherbee SD: From DNA to diversity. Abingdon: Blackwell Science; 200I.

30. Mulley JF, Chiu CH, Holland PW: Breakup of a homeobox cluster after genome duplication in teleosts. Proc Natl Acad Sci USA 2006, 103:10369-10372.

31. Wotton KR, Shimeld SM: Comparative genomics of vertebrate Fox cluster loci. BMC Genomics 2006, 7:27I.

32. Wymore RS, Korenberg JR, Kinoshita KD, Aiyar J, Coyne C, Chen XN, Hustad CM, Copeland NG, Gutman GA, Jenkins NA, Chandy GK: Genomic organization, nucleotide sequence, biophysical properties, and localization of the voltage-gated $\mathrm{K}+$ channel gene KCNA4/KvI.4 to mouse chromosome 2/human IIpI4 and mapping of $\mathrm{KCNCI/Kv3.I}$ to mouse 7/human IIpI4.3 pI5.2 and KCNAI/KvI.I to human I2pI3. Genomics 1994, 20:191-202.
33. Street VA, Tempel BL: Physical Mapping of Potassium Channel Gene Clusters on Mouse Chromosomes Three and Six. Genomics 1997, 44: I 10-1 I7.

34. Roux B: What can be deduced about the structure of Shaker from available data? Novartis Found Symp 2002, 245:84-I0I. discussion I0I-108, I65-108.

35. Doyle DA, Morais Cabral J, Pfuetzner RA, Kuo A, Gulbis JM, Cohen $S L$, Chait BT, MacKinnon R: The structure of the potassium channel: molecular basis of $\mathrm{K}+$ conduction and selectivity. Science 1998, 280:69-77.

36. Anderson PA, Greenberg RM: Phylogeny of ion channels: clues to structure and function. Comp Biochem Physiol B Biochem Mol Biol 200I, 129:17-28.

37. Crow KD, Stadler PF, Lynch VJ, Amemiya C, Wagner GP: The "fishspecific" Hox cluster duplication is coincident with the origin of teleosts. Mol Biol Evol 2006, 23:12I-136.

38. de Souza FS, Bumaschny VF, Low MJ, Rubinstein M: Subfunctionalization of expression and peptide domains following the ancient duplication of the proopiomelanocortin gene in teleost fishes. Mol Biol Evol 2005, 22:24I7-2427.

39. Robinson-Rechavi M, Boussau B, Laudet V: Phylogenetic dating and characterization of gene duplications in vertebrates: the cartilaginous fish reference. Mol Biol Evol 2004, $21: 580-586$.

40. Prohaska SJ, Fried C, Flamm C, Wagner GP, Stadler PF: Surveying phylogenetic footprints in large gene clusters: applications to Hox cluster duplications. Mol Phylogenet Evol 2004, 3I:58I-604.

41. Tagle DA, Koop BF, Goodman M, Slightom JL, Hess DL, Jones RT: Embryonic epsilon and gamma globin genes of a prosimian primate (Galago crassicaudatus). Nucleotide and amino acid sequences, developmental regulation and phylogenetic footprints. J Mol Biol 1988, 203:439-455.

42. Chen WJ, Orti G, Meyer A: Novel evolutionary relationship among four fish model systems. Trends Genet 2004, 20:424-43I.

43. Steinke D, Salzburger W, Meyer A: Novel relationships among ten fish model species revealed based on a phylogenomic analysis using ESTs. J Mol Evol 2006, 62:772-784.

44. RepeatMasker [http://www.repeatmasker.org/cgi-bin/WEBRe peatMasker]

45. Jan LY, Jan YN: Cloned potassium channels from eukaryotes and prokaryotes. Annu Rev Neurosci 1997, 20:91-I23.

46. Kasahara M, Naruse K, Sasaki S, Nakatani Y, Qu W, Ahsan B, Yamada T, Nagayasu Y, Doi K, Kasai Y, Jindo T, Kobayashi D, Shimada A, Toyoda A, Kuroki Y, Fujiyama A, Sasaki T, Shimizu A, Asakawa S, Shimizu N, Hashimoto S, Yang J, Lee Y, Matsushima K, Sugano S, Sakaizumi M, Narita T, Ohishi K, Haga S, Ohta F, et al:: The medaka draft genome and insights into vertebrate genome evolution. Nature 2007, 447:7|4-719.

47. Sidow A: Gen(om)e duplications in the evolution of early vertebrates. Curr Opin Genet Dev 1996, 6:715-722.

48. Wagner GP, Fried C, Prohaska SJ, Stadler PF: Divergence of conserved non-coding sequences: Rate estimates and relative rate tests. Mol Biol Evol 2004, 21:21 I6-2 I2I.

49. Santini S, Boore JL, Meyer A: Evolutionary conservation of regulatory elements in vertebrate hox gene clusters. Genome Res 2003, I3: I III-II 22.

50. Steinke D, Salzburger W, Braasch I, Meyer A: Many genes in fish have species-specific asymmetric rates of molecular evolution. BMC Genomics 2006, 7:20.

51. Fares MA, Byrne KP, Wolfe KH: Rate asymmetry after genome duplication causes substantial long-branch attraction artifacts in the phylogeny of Saccharomyces species. Mol Biol Evol 2006, 23:245-253.

52. Duzhyy DE, Sakai Y, Sokolowski BH: Cloning and developmental expression of Shaker potassium channels in the cochlea of the chicken. Brain Res Mol Brain Res 2004, I $21: 70-85$

53. Mayor C, Brudno M, Schwartz IR, Poliakov A, Rubin EM, Frazer KA Pachter LS, Dubchak I: VISTA : visualizing global DNA sequence alignments of arbitrary length. Bioinformatics 2000 , 16:1046-1047.

54. Hedges SB, Kumar S: Genomic clocks and evolutionary timescales. Trends Genet 2003, 19:200-206.

55. Fried C, Prohaska S, Stadler PF: Exclusion of repetitive DNA elements from gnathostome Hox clusters. J Exp Zool B Mol Dev Evol 2004, 302:165-173. 
56. Oliver B, Misteli T: A non-random walk through the genome. Genome Biol 2005, 6:2। 4.

57. Hall TA: BioEdit: a user-friendly biological sequence alignment editor and analysis program for Windows 95/98/NT. Nucleic Acids Symp Ser 1999, 4 1:95-98.

58. Abascal F, Zardoya R, Posada D: ProtTest: selection of best-fit models of protein evolution. Bioinformatics 2005, 21:2 104-2105.

59. Guindon S, Gascuel O: A simple, fast, and accurate algorithm to estimate large phylogenies by maximum likelihood. Syst Biol 2003, 52:696-704.

60. Huelsenbeck JP, Ronquist F: MRBAYES: Bayesian inference of phylogenetic trees. Bioinformatics 200I, 17:754-755.

Publish with Bio Med Central and every scientist can read your work free of charge

"BioMed Central will be the most significant development for disseminating the results of biomedical research in our lifetime. "

Sir Paul Nurse, Cancer Research UK

Your research papers will be:

- available free of charge to the entire biomedical community

- peer reviewed and published immediately upon acceptance

- cited in PubMed and archived on PubMed Central

- yours - you keep the copyright

Submit your manuscript here:

http://www.biomedcentral.com/info/publishing_adv.asp
BiolMedcentral 